\title{
Dynamic Multicomponent Reactions Directed Self-assembled G-quadruplex Inherent Antibacterial Hydrogel
}

\author{
Tanmay Rit, ${ }^{\dagger}$ Tapas Ghosh, ${ }^{\dagger}$ Mukesh K. Patidar, ${ }^{\dagger}{ }^{\dagger}$ Apurba K. Das ${ }^{\dagger *}$ \\ ${ }^{\dagger}$ Department of Chemistry, Indian Institute of Technology Indore, Indore 453552, India \\ \aharaja Ranjit Singh College of Professional Sciences, Indore 452001, India \\ Email: apurba.das@iiti.ac.in
}

\begin{abstract}
Now-a-days inherent antibacterial hydrogels have gained significant attention due to their utilization against infectious bacteria. Herein, we focus on the development of injectable, self-healable, dynamic, G-quadruplex hydrogel with inherent antibacterial activity. The dynamic self-assembled hydrogel is constructed upon multicomponent reactions (MCR) among guanosine, 2-formylphenylboronic acid and amino acid/peptides in presence of potassium ions. The role of amino acid/peptides toward the formation of G-quadruplex hydrogel is studied in details. G-quadruplex structure is formed via the $\pi$ - $\pi$ stacking of Gquartets. The formation of G-quadruplex is investigated by thioflavin $\mathrm{T}$ binding assay, $\mathrm{CD}$ spectroscopy and PXRD. The formation of the dynamic imino-boronate bond in the hydrogels is well characterised by temperature dependent ${ }^{11} \mathrm{~B}$ NMR (VT-NMR) and FT-IR spectroscopy. Furthermore HR-TEM images and rheological experiments reveal the fibrillar networks and the viscoelastic property of the hydrogels. The presence of the dynamic iminoboronate ester bonds makes the hydrogel injectable and self-healable in nature. These dynamic G-quadruplex hydrogels show potential antibacterial activity against a series of Gram positive and Gram negative bacteria. Such injectable and self-healable hydrogels could be used in various applications in the field of biomedical science.
\end{abstract}




\section{Introduction}

Bacterial infections have become a great threat for mankind and the infection rate is increasing day by day. Many antibiotics have been developed to combat bacterial infection, however, the microbes have evaluated themselves to become antibiotics resistant. ${ }^{1}$ Recently antibacterial biomaterial like hydrogel has paved a way to fight infectious bacteria., Antibacterial activity of any hydrogel can be achieved by the incorporation of nanoparticles and antibiotics within the hydrogel. ${ }^{2}$ Silver nanoparticles incorporated hydrogel is a wellknown example of an antibacterial hydrogel. ${ }^{4}$ Silver ions $\left(\mathrm{Ag}^{+}\right)$stabilized hydrogel has also been reported as an antibacterial hydrogel. ${ }^{5}$ However, there are few limitations of the abovementioned hydrogels. Antibiotics loaded hydrogels ${ }^{6,7}$ are becoming incapable towards the bacterial infection due the antibiotic resistance of bacteria. ${ }^{1}$ Silver incorporated hydrogels have few side effects such as irritation in skin or inflammation. ${ }^{8}$ Therefore, to overcome these limitations, researchers are focusing on the development of biocompatible, biodegradable hydrogels with inherent antibacterial activity. ${ }^{9}$ Polymer based antibacterial hydrogels ${ }^{10}$ have different drawbacks as the synthesis and purification of these compounds are a tedious job. Hence, low molecular weight gelators (LMWGs) ${ }^{11,12}$ such as nucleobase ${ }^{13,14}$ and peptide ${ }^{15}$ based inherent antibacterial hydrogel could be an efficient candidate to resolve this problem. Peptides can undergo spontaneous self-assembly in appropriate conditions to form threedimensional (3D) nanofibrillar networks. ${ }^{16}$ Moreover, the potentiality of peptide based hydrogels in various fields such as drug delivery, tissue engineering and bio-imaging has acquired admiration in recent years. ${ }^{17-19}$ Apart from this, being biocompatible as well as biodegradable, nucleobases are the promising candidates for the development of versatile hydrogels. ${ }^{20}$ Guanosine, one of the nucleosides, has been used as a self-assembling potential candidate for the development of LMWGs. ${ }^{21}$ In guanosine, four guanosine units furnish a Gquartet moiety in presence of cations such as $\mathrm{K}^{+}, \mathrm{Na}^{+}$with the help of intermolecular 
Hoogsteen type H-bonding interactions which further self-assemble into G-quadruplex structures via $\pi-\pi$ stacking interaction and leads to the formation of nanofibrous hydrogel. ${ }^{22,23,24}$ Davis et al have shown the formation of metal ion induced G-quartet hydrogels and alternation in their characteristic features on changing the metal ions. ${ }^{22}$

In recent years, dynamic covalent bond mediated hydrogels have gained significant attentions for their stimuli-responsive (temperature, pressure and $\mathrm{pH}$ ) and reversible nature. ${ }^{25}$ Among various dynamic bonds, boronate ester and imine bond directed hydrogels and their applications in the field of biomedical science have been explored. ${ }^{26-30}$ The rate of formation of cyclic boronate ester is enhanced by the condensation between ortho carbonyl (aldehyde or ketone) and amine. ${ }^{31}$ Nitrogen atom of imine bond donates its lone pair of electrons to the boron center through a dative bond facilitating the formation of tetrahedral boronate ion. ${ }^{32}$ Exploiting this behaviour of boronic acid, hydrogel based optical diffuser has been reported. ${ }^{33}$ The boronic acid based hydrogels have been utilized for three-dimensional (3D) cell culture. ${ }^{34}$ The $\mathrm{pH}$ dependent nature of boronate ester bond has been explored in the delivery of anticancer drug-loaded nanoparticles. ${ }^{35}$ On the other hand, primary amine can form dynamic imine bond by reacting with aldehyde at relatively basic $\mathrm{pH}^{36}$ The imine hydrogel has been reported for modulating the degradation behaviour of $3 \mathrm{D}$ cell culture for the controlled release of cells. ${ }^{37}$ Recently, $\mathrm{pH}$ responsive drug delivery applications have been employed by incorporating such dynamic bonds in hydrogel system. ${ }^{38,39}$ Imino-boronate bond directed hydrogels are capable of showing greater self-healing and stimuli responsive behaviour due to the presence of double dynamic covalent bond..$^{40,41}$

Here, we focus on the development of multicomponent reaction (MCR) among guanosine, peptide and boronic acid directed injectable, self-healable, stimuli responsive inherent antibacterial hydrogel. The hydrogel is constructed upon the formation of dynamic covalent bond and non-covalent interaction among guanosine, 2-FPBA and peptides (or amino acid) in 
$\mathrm{KOH}$ solution. We have evaluated the antibacterial activity of this dynamic imino-boronate bond assisted G-quadruplex hydrogels against both Gram positive as well as Gram negative bacteria.

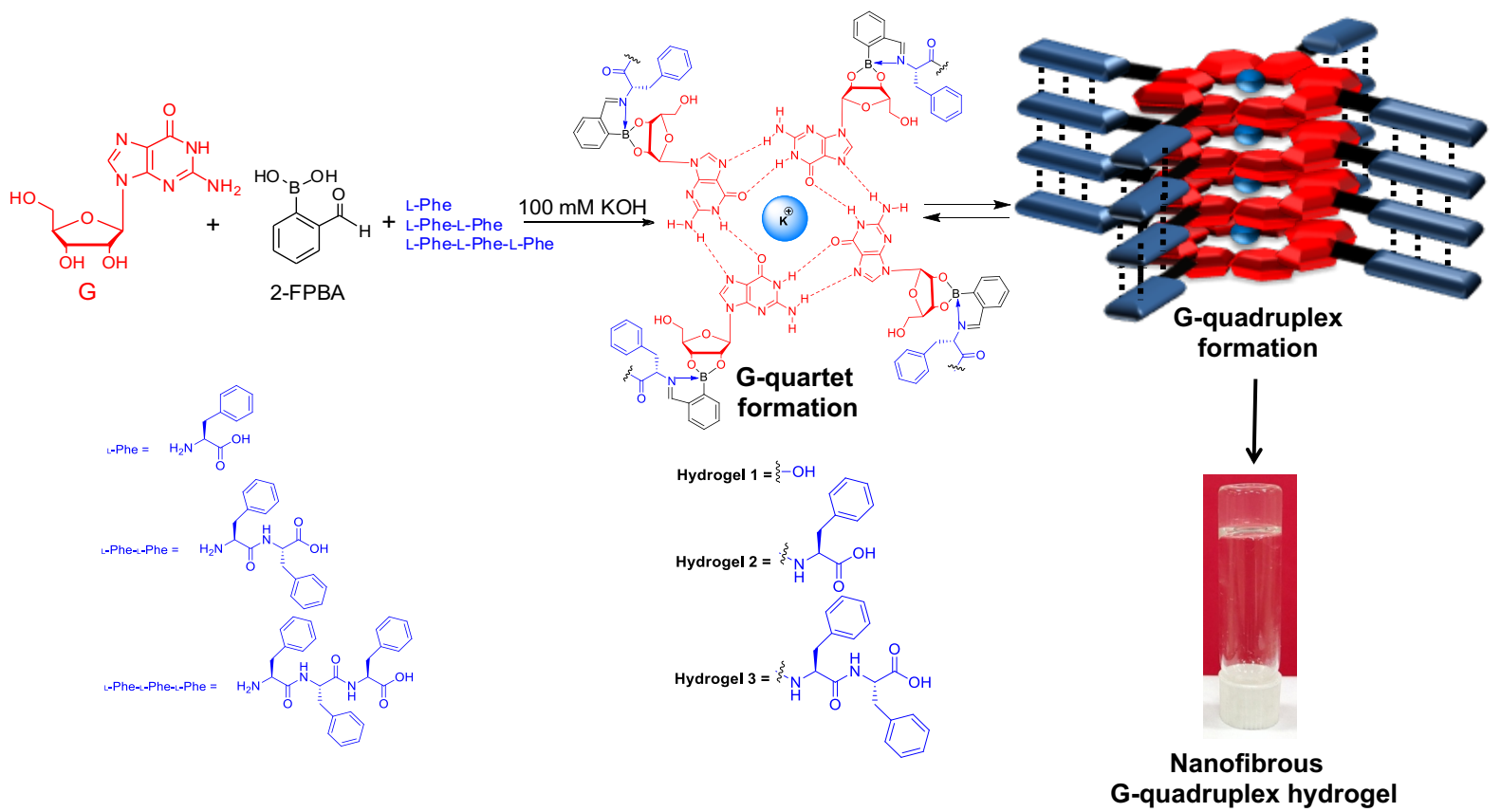

Figure 1. The schematic representation of the formation of MCR directed dynamic Gquadruplex hydrogel $(50 \mathrm{mM})$ in presence of $100 \mathrm{mM} \mathrm{KOH}$ solution. First, the formation of G-quartet takes place and finally G-quadruplex structured is formed via the $\pi-\pi$ stacking interaction.

\section{Results and Discussion}

In general, boronic acid undergoes condensation reaction with cis-1,2- and 1,3-diol at a $\mathrm{pH}$ greater or equal to $\mathrm{pKa}$ of boronic acid to form stable 5-membered and 6-membered cyclic boronate esters respectively. ${ }^{30}$ Guanosine, 2-FPBA and individual amino acid (Lphenylalanine; L-Phe) or peptides (L-Phe-L-Phe and L-Phe-L-Phe-L-Phe) were taken in a glass vial at a molar ratio of 1:1:1 $(50 \mathrm{mM} / 50 \mathrm{mM} / 50 \mathrm{mM}$ respectively) and dissolved it in 100 $\mathrm{mM} \mathrm{KOH}$ solution. The glass vial was then sonicated for $5 \mathrm{~min}$ followed by heating at $80{ }^{\circ} \mathrm{C}$ 
till the solution became transparent, left to cool it down to room temperature. A transparent, injectable and self-healable hydrogel was obtained in all cases. The formation of hydrogels was checked by the inverted vial method (Figure S1). Intermolecular hydrogen bonding among four guanosine molecules and electrostatic interaction between $\mathrm{K}^{+}$ion and guanosines give a G-quartet structure. These G-quartets further self-assemble via $\pi-\pi$ stacking interaction and give rise to a cylindrical G-quadruplex (G4) structure. 2-FPBA forms a 5-membered dynamic boronate ester upon reaction with the cis-1,2-diol of the ribose sugar of the guanosine. On the other hand dynamic imine bond formation takes place between the primary amine group of L-Phe / L-Phe-L-Phe / L-Phe-L-Phe-L-Phe and the formyl group of 2-FPBA. These two dynamic covalent bonds along with the non-covalent interactions (H-bonding, $\pi-\pi$ stacking and electrostatic interaction) develop 3D nanofiber networks in which water molecules get encapsulated and form the hydrogel (Figure 1). All the three hydrogels are fluorescent in nature and show emission peak at $457 \mathrm{~nm}$ (Figure S1). The intensity of the peak rises from hydrogel $\mathbf{1}$ to $\mathbf{2}$ and $\mathbf{3}$ gradually as the number of phenyl rings in the hydrogels increases, which clearly indicates the enhancement of $\pi-\pi$ stacking interaction between the phenyl rings.
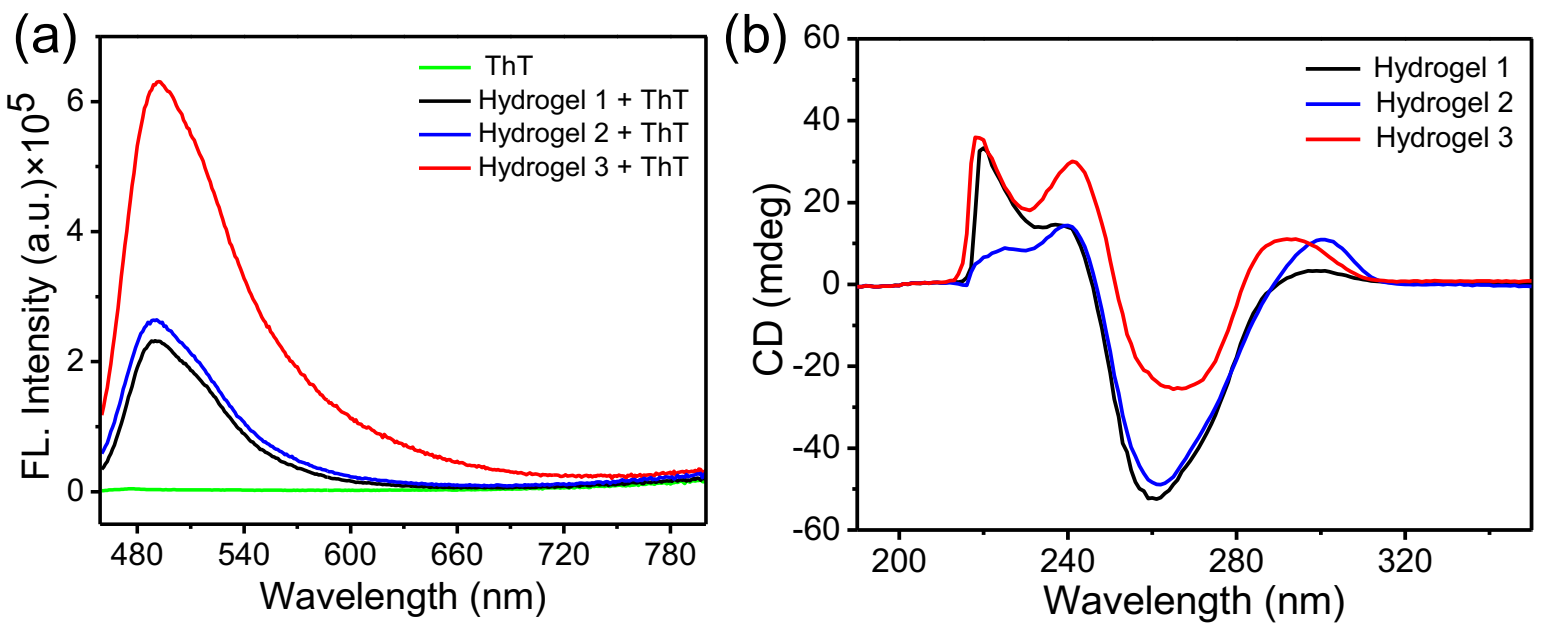

Figure 2. Determination of G-quadruplex structures. (a) Fluorescence spectra of ThT incorporated hydrogels $\left(\lambda_{\mathrm{ex}}=450 \mathrm{~nm}\right)$ and (b) CD spectra of diluted $(5 \mathrm{mM})$ hydrogels. 
The formation of G-quadruplex architecture was investigated by using the fluorophore Thioflavin T (ThT) binding assay. ${ }^{42}$ In this experiment, ThT solution $(1 \mathrm{mM}, 100 \mu \mathrm{L})$ was added to each hydrogels during the preparation of hydrogels. The fluorescence spectra of the ThT incorporated hydrogels show intense peaks at $490 \mathrm{~nm}$. However, only ThT does not show any emission peak at $490 \mathrm{~nm}$. The ThT incorporated hydrogel shows emission peak at $490 \mathrm{~nm}$ because it is stacked between two G-quartet via $\pi$ - $\pi$ stacking interaction. Hence, it shows a peak of higher intensity as well as peak shifting. The increase in fluorescence intensity of ThT loaded hydrogels clearly confirms the formation of G-quadruplex-like structures in the hydrogel. As depicted from Figure 2, ThT incorporated hydrogel 3 shows the highest intensity peak followed by hydrogel 2 and 1. This observation indicates higher stacking of ThT in the hydrogel takes place due to the presence of greater number of phenyl rings in hydrogel 3. Hence, these results further support the previous fluorescence results.

Circular Dichroism (CD) is one of the most efficient tools to investigate the secondary interaction $^{43}$ in a supramolecular system. For hydrogel 1, two positive peaks at 240, $296 \mathrm{~nm}$ and one negative peak at $260 \mathrm{~nm}$ are observed. The CD peaks at 296 and $260 \mathrm{~nm}$ indicate the head to head stacking interaction between G-quartets whereas peaks at 240 and $260 \mathrm{~nm}$ indicate the head to tail stacking interaction. Another peak centered at $222 \mathrm{~nm}$ is attributed to the presence of phenyl ring of 2-FPBA. Hydrogel 2 shows positive peaks at 240, $300 \mathrm{~nm}$ and a negative peak at $260 \mathrm{~nm}$ indicating the head to head as well as head to tail stacking arrangement of G-quartets. Similarly, hydrogel 2 shows an absorption peak at $220 \mathrm{~nm}$ which depicts the presence of the phenyl ring in the hydrogel. Three positive peaks at 220, 240, 290 $\mathrm{nm}$ and one negative peak at $266 \mathrm{~nm}$ are obtained in case of hydrogel 3 . The peak observation is similar to the other two hydrogels, which indicates the presence of head to head as well as head to tail stacking of G-quartets in all the three hydrogels (Figure 2). ${ }^{44,45}$ The Powder X-ray Diffraction (PXRD) pattern of all three hydrogels show a major peak at $2 \theta$ 
value of $26.8^{\circ}$ corresponding to the distance $(d)$ of $3.3 \AA$ indicating the distance between two adjacent stacked G-quartets (Figure 3). ${ }^{46,47}$ The PXRD patterns of all the building blocks of hydrogels have been recorded separately for the comparison with the pattern of the hydrogels (Figure S2). The absence of the peak at $26.8^{\circ}$ for all the building blocks clearly indicates that G-quadruplex is only formed in the hydrogels.
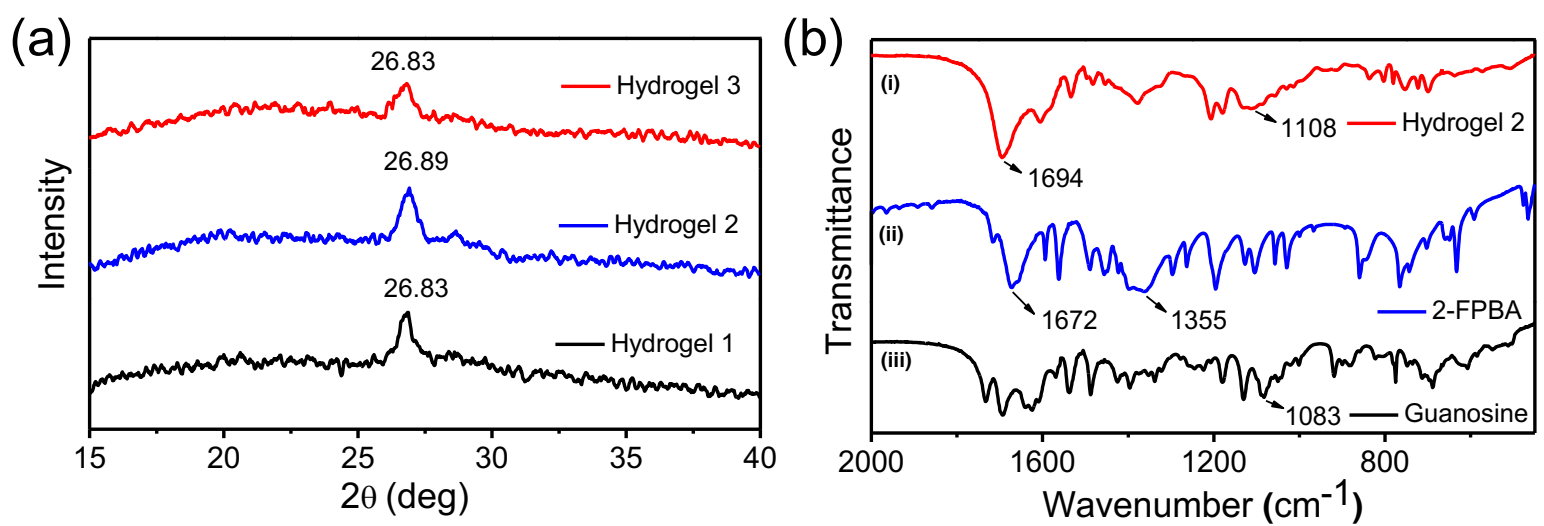

Figure 3. (a) PXRD patterns of dried hydrogels indicate the formation of G-quadruplex architectures in the hydrogels. (b) FT-IR spectra of (i) dried hydrogel 2, (ii) 2-FPBA and (iii) guanosine.

The formation of boronate ester and imine bond in the hydrogel is investigated with the help of Nuclear Magnetic Resonance (NMR) Spectroscopy $\left({ }^{11} \mathrm{~B}\right.$ NMR) and Fourier Transform Infrared Spectroscopy (FT-IR). The formation of intramolecular boronate ester bond has been investigated with the help of ${ }^{11} \mathrm{~B}$ NMR spectroscopy. 2-FPBA shows a peak at $30.85 \mathrm{ppm}$ corresponding to the $\mathrm{B}-\mathrm{O}-\mathrm{H}$ bond ${ }^{39}$ whereas in presence of $\mathrm{KOH}$ solution this peak is shifted to $7.56 \mathrm{ppm}$ (Figure S3). This observation signifies the formation of intramolecular boronate ester (B-O-C) bond. The formation of dynamic intermolecular boronate ester bond between guanosine and 2-FPBA was checked by the variable temperature ${ }^{11} \mathrm{~B}$ NMR (VT-NMR) at various temperatures $\left(15,30,40,50,60\right.$ and $\left.80{ }^{\circ} \mathrm{C}\right)$. Intramolecular boronate ester bond starts to break and the generation of intermolecular boronate ester bond takes place as the 
temperature increases from 15 to $80{ }^{\circ} \mathrm{C}$ gradually. The appearance of a new peak at 12.08 ppm in ${ }^{11} \mathrm{~B}$ NMR spectra clearly indicates the formation of intermolecular boronate ester bond while the intensity of peak at $7.78 \mathrm{ppm}$ decreases indicating the rupture of intramolecular boronate ester bond in 2-FPBA (Figure S3). The free 2-FPBA shows chemical shift at 30.85 ppm signifying the presence of B-O-H bond whereas the hydrogel 2 displays the upfield chemical shift at $12.08 \mathrm{ppm}$ suggesting the formation of B-O-C bond in hydrogel. ${ }^{39}$ The intensity of the peak at $12.08 \mathrm{ppm}$ is enhanced as the temperature increases from 15 to $80{ }^{\circ} \mathrm{C}$, which indicates the formation of intermolecular boronate ester bond between $\mathrm{G}$ and FPBA. This dynamic boronate ester bond is the key component of this dynamic G-quadruplex hydrogels. ${ }^{48,49}$ The FT-IR spectra of 2-FPBA shows a stretching frequency peak at $1355 \mathrm{~cm}^{-1}$ corresponding to the $\mathrm{B}-\mathrm{O}-\mathrm{H}$ bond. The appearance of a peak at $1108 \mathrm{~cm}^{-1}$ for all the dried hydrogels dictates the formation of $\mathrm{B}-\mathrm{O}-\mathrm{C}$ bond. Furthermore, the stretching frequency of guanosine C-O-H bond at $1084 \mathrm{~cm}^{-1}$ disappears in all the hydrogels. A peak at $1672 \mathrm{~cm}^{-1}$ for 2-FPBA due to the presence of aldehyde $\mathrm{C}=\mathrm{O}$ shifts to $1694 \mathrm{~cm}^{-1}$ for hydrogel 2 signifying the formation of imine bond between aldehyde group of 2-FPBA and amine group of amino acid (L-Phe) or peptides (L-Phe-L-Phe and L-Phe-L-Phe-L-Phe). ${ }^{38,39}$ The morphological analysis of the hydrogels was performed using HR-TEM. The nanofibrillar networks are observed in all the hydrogels, suggesting the formation of nanofibrillar structures via the $\pi-\pi$ stacking interaction between G-quartet architectures. The nanofibrillar networks of hydrogel 2 and $\mathbf{3}$ are presented in Figure 4 . The width of the nanofibers are in the range of 15-20 nm for hydrogel $\mathbf{1}$ and 20-25 $\mathrm{nm}$ for hydrogel 2 and 3 respectively. 

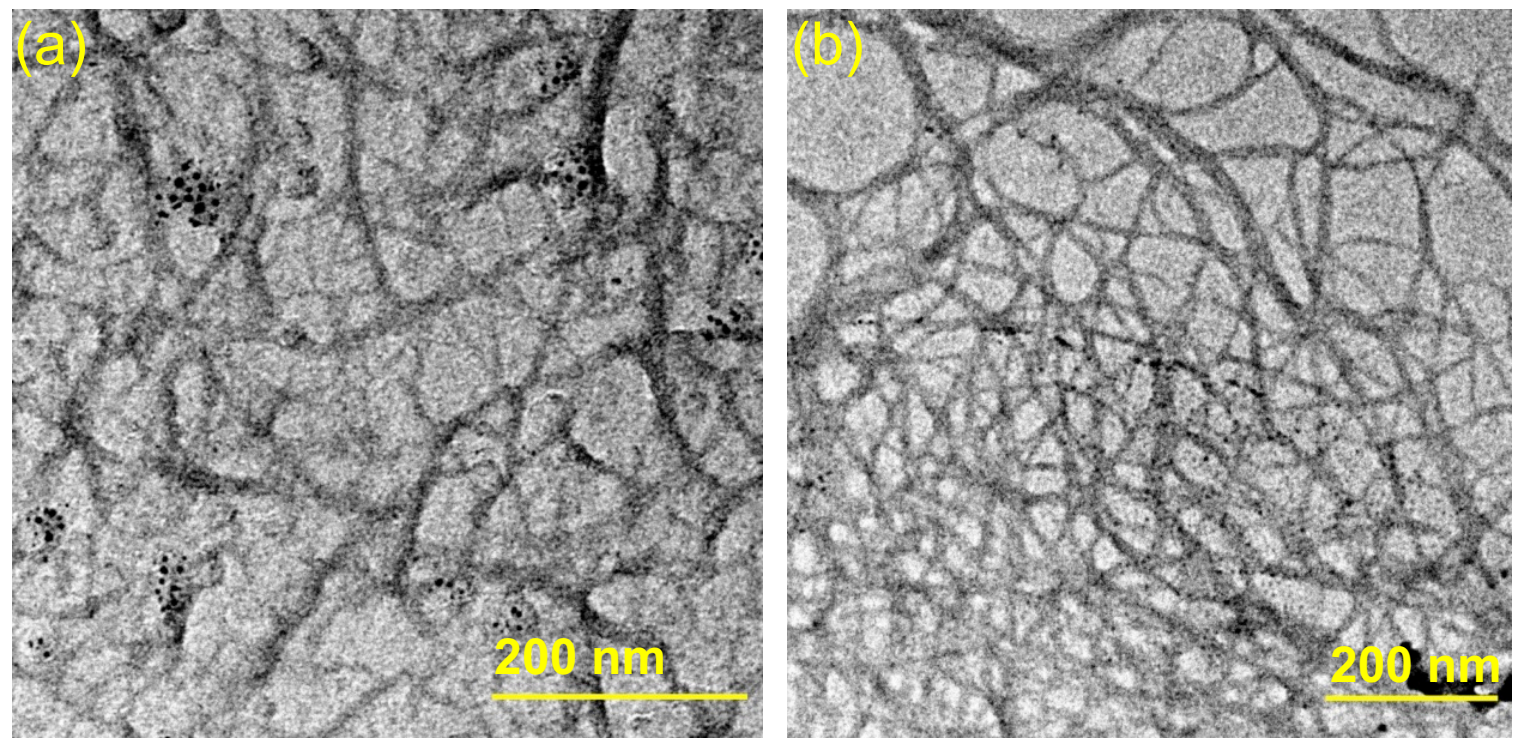

Figure 4. TEM images of (a) hydrogel 2 and (b) hydrogel 3 depict the three-dimensional nanofibrillar structure of hydrogels.

Viscoelastic properties of all three hydrogels were evaluated by the rheological experiments. Amplitude sweep experiments for all the hydrogels were carried out at constant frequency rate of $1 \mathrm{rad} \mathrm{s}^{-1}$. All hydrogels show higher storage modulus $\left(\mathrm{G}^{\prime}\right)$ than that of loss modulus $\left(G^{\prime \prime}\right)$ value at $0.1-10 \%$ shear strain, specifying the gel state of all the samples. The $G^{\prime}$ value gradually decreases above the shear strain of $10 \%$ intersecting with $G^{\prime \prime}$ value at a certain point, which dictates the beginning of the sol state. The intersection points of $\mathrm{G}^{\prime}$ and $\mathrm{G}^{\prime \prime}$ for hydrogel 1, 2 and $\mathbf{3}$ are at 396, 50 and 127\% of deformation strain, respectively (Figure S4). Furthermore, frequency sweep experiments were performed at constant shear strain $(1 \%)$ keeping the frequency range of $0.5-100 \mathrm{rad}^{-1}{ }^{-1}$. All the hydrogels exhibit higher storage modulus value $\left(\mathrm{G}^{\prime}\right)$ than the storage modulus value $\left(\mathrm{G}^{\prime \prime}\right)$ throughout the experiment justifying the viscoelastic nature of the hydrogels. As depicted from Figure 5, the hydrogel 3 exhibits higher $\mathrm{G}^{\prime}$ value of $806 \mathrm{~Pa}$ followed by hydrogel 2 of $485 \mathrm{~Pa}$ and hydrogel 1 of $35 \mathrm{~Pa}$. These observations clearly indicate that hydrogel $\mathbf{3}$ is more robust than hydrogels $\mathbf{1}$ and $\mathbf{2}$, resulting from the higher degree of $\pi-\pi$ stacking interaction in hydrogel 3. As hydrogel 3 is more 
mechanically robust, it has been chosen to perform dynamic step-strain sweep experiment to determine the thixotropic behaviour of the hydrogel (Figure 5b). The experiment was performed at constant angular frequency of $10 \mathrm{rad} \mathrm{s}^{-1}$ with alternative lower (1\%) and higher $(100 \%)$ strains throughout the experiment for $900 \mathrm{~s}$. The storage modulus $\left(\mathrm{G}^{\prime}\right)$ is higher than that of loss modulus $\left(\mathrm{G}^{\prime \prime}\right)$ at lower strain $(1 \%)$ depicting the gel state, whereas reverse is observed at higher strain (100\%) dictating the sol state of the hydrogel. Upon increasing the strain from 1 to $100 \%$, the fibrillar networks in hydrogel were disrupted and the gel transformed into solution. In addition, the reformation of gel took place as soon as the strain was decreased from 100 to $1 \%$. This observation clearly suggests the rapid sol to gel and gel to sol transformation, which takes place under the applied strain.
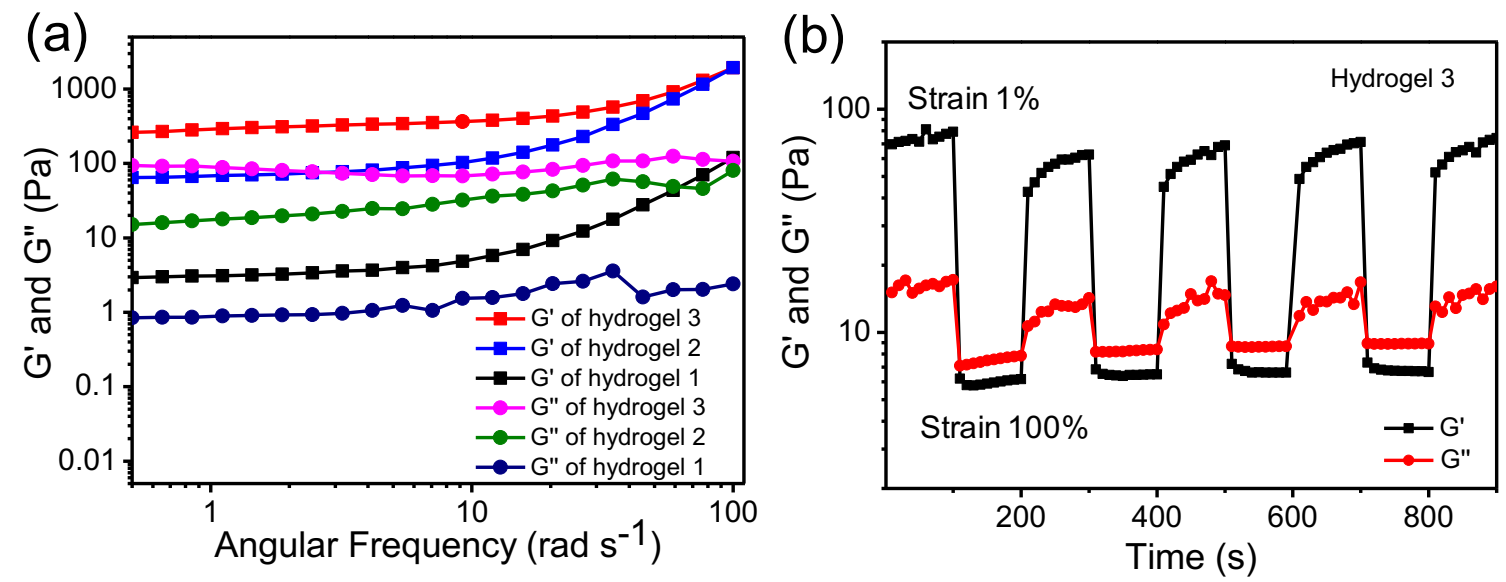

Figure 5. Rheological experiments. (a) Frequency sweep analysis of all the hydrogels at a constant strain of 1\%. (b) Dynamic step-strain sweep experiment of hydrogel 3 at a constant angular frequency of $10 \mathrm{rad} \mathrm{s}^{-1}$.

Along with the thixotropic nature, hydrogel 3 shows quite satisfactory result in injectability test (Figure 6). Hydrogel 3 was doped with a dye during the preparation and injected through a needle to check the thixotropic nature of the hydrogel. After injecting into a vial, the hydrogel retains its gel state very rapidly, which was examined by inverting the glass vial. At 
the time of injection, the hydrogel was transformed into its sol state due to the operating pressure by the syringe, as soon as it came out of the needle it retained the gel state. Hydrogels consist of dynamic covalent bonds and non-covalent interactions, which are capable of showing the self-healing feature. The dynamic nature of both the covalent bond and non-covalent interaction is the major reason behind the self-healing property. The propensity to reform the covalent bond or re-establish the non-covalent interaction among the components to maintain the dynamic equilibrium in the system gives rise to the self-healing characteristics. Two pieces of hydrogel $\mathbf{3}$ was kept on a petridish at room temperature, one of the pieces was doped with methyl red dye and the other one was kept untreated. After 30 min, self-healing process started and the self-healing process across the junction point was observed by diffusion of orange colour to the colourless piece. Finally fully self-healed hydrogel was observed after 6h (Figure 6).

(a)

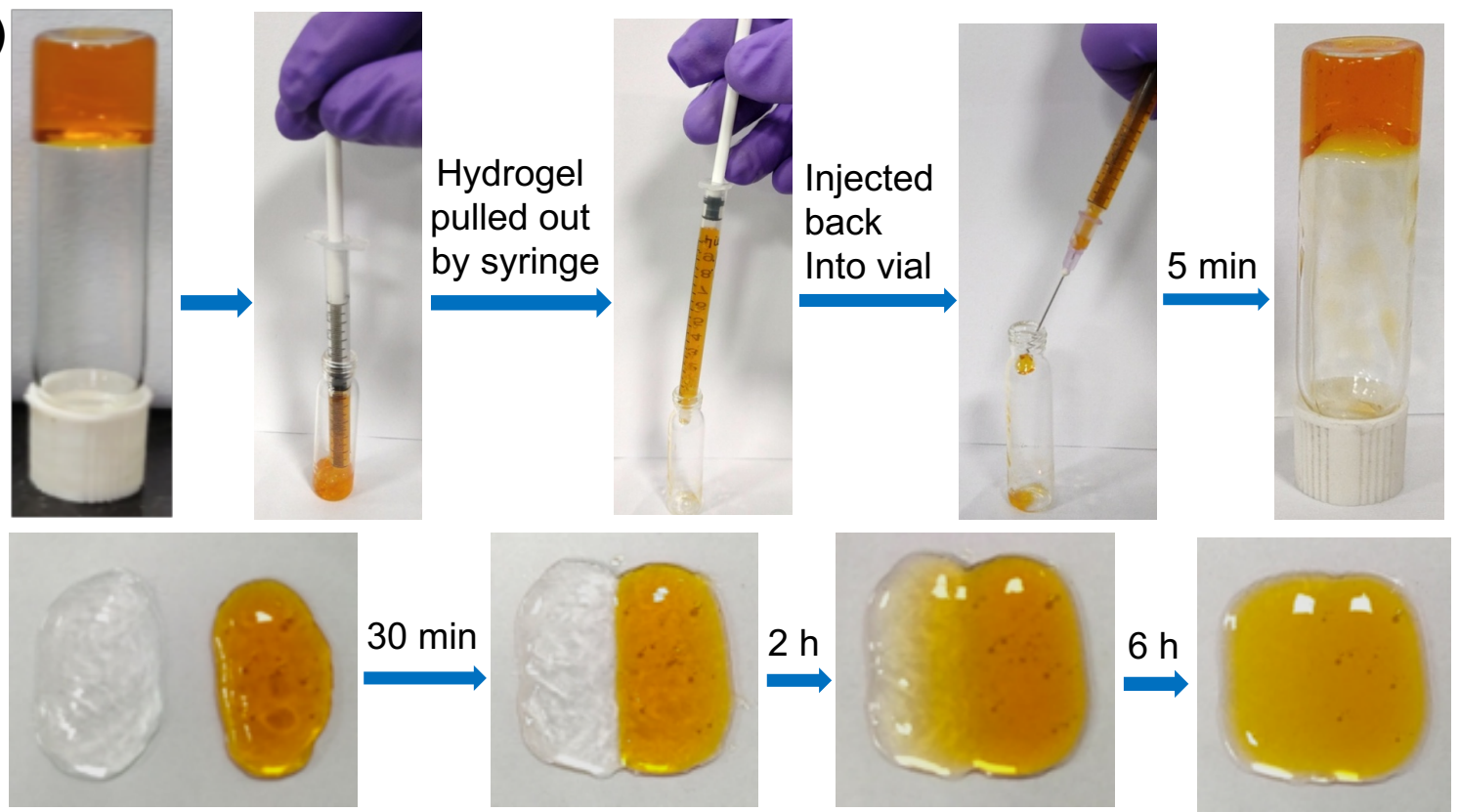

Figure 6. (a) Hydrogel 3 was injected through a $24 \mathrm{G}$ needle to check the injectibility of the hydrogel; (b) Two pieces of hydrogel 3 were kept together to check the self-healing ability. 
Furthermore, stimuli responsiveness of the hydrogel 3 was checked by heating and changing the $\mathrm{pH}$ of the hydrogel (Figure S5). Hydrogel 3 was heated at $80{ }^{\circ} \mathrm{C}$ and it turned into a solution. At the time of heating due to the disruption of the nanofiber networks, the encapsulated water molecules become free and the hydrogel is converted into solution. As the temperature of the solution decreases, the reformation of nanofibers takes place and the gel formation is observed. Along with thermo-responsiveness, $\mathrm{pH}$ dependency of the hydrogel 3 was investigated. $0.5 \mathrm{~N} \mathrm{HCl}(50 \mu \mathrm{L})$ was added dropwise to the hydrogel 3 . The gel turned into white solution instantly signifying the rupture of dynamic imino boronate ester, as well as the H-bonding present in G-quartet. Then $0.5 \mathrm{~N} \mathrm{NaOH}(100 \mu \mathrm{L})$ was added dropwise to maintain the relative basic $\mathrm{pH}$. The solution became transparent. The transparent solution kept undisturbed for few hours resulting into the formation of hydrogel. At basic $\mathrm{pH}$, the reformation of imino boronate ester bond takes place as well as the deprotonation of guanosine G-quartet is formed again via H-bonding. Hence, the hydrogel reveals its stimuli responsive behaviour significantly.

Hydrogel with inherent antibacterial activity has become a potential candidate against bacterial infection. Therefore, all the hydrogels were utilized to investigate the inherent antibacterial activity against a series of Gram negative (Proteus mirabilis, Escherichia coli, Salmonella typhi) and Gram positive bacteria (Streptococcus pneumonie, Staphylococcus aureus, Bacillus subtilis). Antibacterial efficacy was evaluated by the diffusion plate method. ${ }^{50}$ Hydrogels 2 and $\mathbf{3}$ show satisfactory antibacterial activity against all the Gram positive and Gram negative bacteria whereas hydrogel 1 shows antibacterial activity against Streptococcus pneumonie (Figure 7). The zone of inhibition index values have been calculated (Table 1) and these indicate that hydrogels 2 and $\mathbf{3}$ are more effective than hydrogel 1. Furthermore, the antibacterial activity of all the hydrogels (1,2 and 3) was checked by optical density $\left(\mathrm{OD}_{625}\right)$ method. Hydrogel containing bacterial solutions show 
lower absorbance as compared to the control solution (Figure S6) for both Gram positive and Gram negative bacteria. This observation clearly indicates the antibacterial activity of the hydrogels. Hydrogel 1 containing bacterial solution shows higher absorbance as compared to hydrogels 2 and 3 containing bacterial solutions (Figure S6). This observation depicts that hydrogel 3 is most effective against bacteria (Gram positive and Gram negative) followed by hydrogels 2 and 1.

(a)

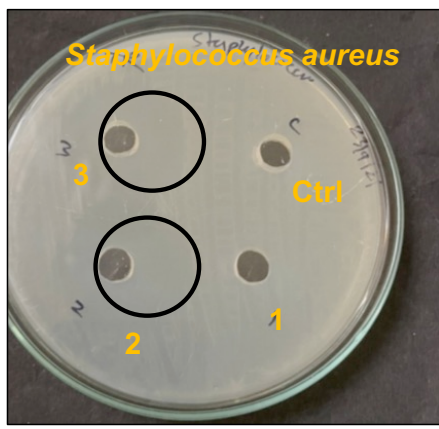

(d)

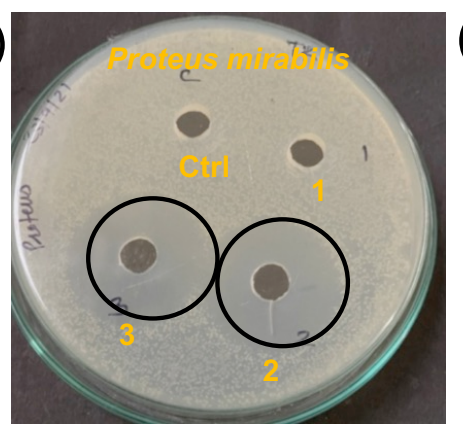

(b)

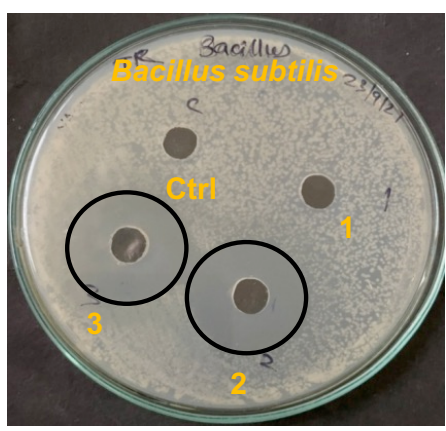

(e)

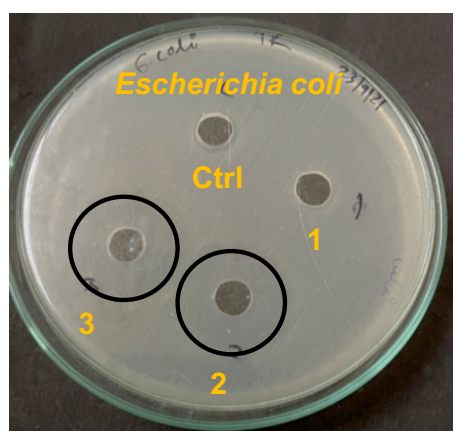

(c)

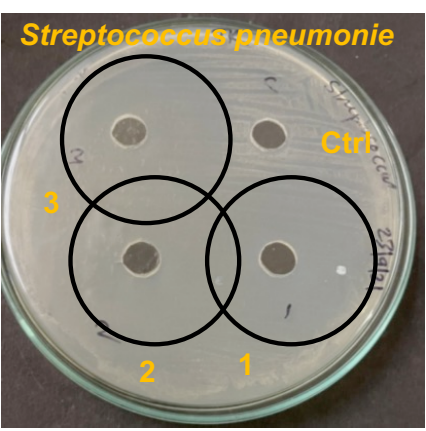

(f)

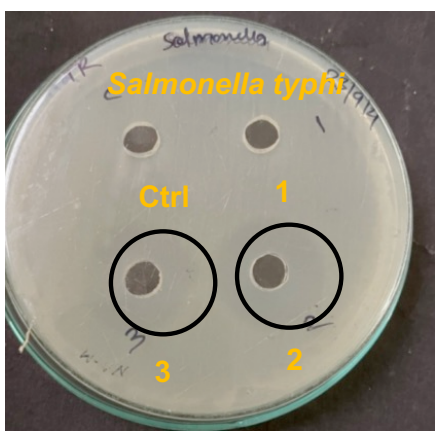

Figure 7. Photographs of agar plate after applying hydrogel 1 (1), hydrogel 2 (2) and hydrogel 3 (3) against Gram positive bacteria (a) Staphylococcus aureus, (b) Bacillus subtilis, (c) Streptococcus pneumonie and Gram negative bacteria (d) Proteus mirabilis, (e) Escherichia coli, (f) Salmonella typhi. 
Table 1. Zone of inhibition index calculated after applying hydrogels over the agar plates of bacteria

\begin{tabular}{|c|l|c|c|c|}
\hline \multirow{2}{*}{ S1. } & \multicolumn{2}{|c|}{ Bacteria } & \multicolumn{3}{|c|}{ Zone of Inhibition Index } \\
\cline { 3 - 5 } & & Hydrogel 1 & Hydrogel 2 & Hydrogel 3 \\
\hline 1 & Streptococcus pneumonie & 2.81 & 2.81 & 2.81 \\
\hline 2 & Staphylococcus aureus & - & 2.00 & 2.18 \\
\hline 3 & Bacillus subtilis & - & 3.56 & 3.62 \\
\hline 4 & Proteus mirabilis & - & 3.50 & 3.68 \\
\hline 5 & Escherichia coli & - & 2.93 & 3.06 \\
\hline 6 & Salmonella typhi & - & 3.12 & 3.00 \\
\hline
\end{tabular}

ac ' indicates that hydrogel does not show antibacterial activity after utilizing on bacteria.

The difference in antibacterial activity of three hydrogels is observed due to the difference in hydrophobicity in molecular self-assembly. The number of phenyl ring at the side chain of amino acid/peptide increases from hydrogel 1 to hydrogel 2 to hydrogel 3, which indicates the increase in hydrophobicity. Moreover, the damage created by the hydrogels at the membrane of bacteria and the disruption of membrane causes the death of bacteria, which are clearly observed from SEM images (Figure S7). ${ }^{51}$

\section{Conclusion}

In the present study, we have successfully developed dynamic, stimuli responsive, selfhealing G-quadruplex hydrogel with inherent antibacterial activity. The hydrogels (2 and 3) displayed astonishing inherent antibacterial property against both the Gram positive (Streptococcus pneumonie, Staphylococcus aureus, Bacillus subtilis) and Gram negative (Proteus mirabilis, Escherichia coli, Salmonella typhi) bacteria. The synergistic effect of peptide conjugated G-quadruplex hydrogel was responsible for the inherent antibacterial 
activity. The formation of G-quadruplex architecture was characterised by ThT binding assay, CD spectroscopy and PXRD. The dynamic imino-boronate ester bond formation was investigated by FT-IR spectroscopy. HR-TEM images described the formation of nanofibrillar network in the hydrogels. The frequency sweep and dynamic strain-step experiments revealed the viscoelastic and thixotopic nature of the hydrogels. Furthermore, these hydrogels showed efficient self-healing and injectable behaviour which have an enormous application in the field of biomedical science. The hydrogels $\mathbf{2}$ and $\mathbf{3}$ were more efficient antibacterial agents as compared to the hydrogel 1. In future, hydrogel 2 and 3 could be used as efficient antibacterial biomaterials in in vitro and in vivo applications.

\section{Acknowledgement}

A.K.D. sincerely acknowledges CSIR, New Delhi, Government of India (Project no. 01(2936)/18/EMR-II) for financial support. A.K.D. and M.K.P. also acknowledge the Science and Engineering Research Board (SERB), Department of Science and Technology, Govt. of India under the scheme TARE (Grant No. TAR/2020/000399) for the financial support. SIC, IIT Indore is acknowledged for providing instrumentation support. T.R. sincerely acknowledges CSIR Fellowship, Government of India for his doctoral fellowship. T.G. is indebted to DST-Inspire Fellowship, Government of India. SAIF, IIT Bombay is acknowledged for TEM facility.

\section{References}

1. Prestinaci, F.; Pezzotti, P.; Pantosti, A. Antimicrobial Resistance: A Global Multifaceted Phenomenon. Pathog. Global Health. 2015, 109, 309- 18.

2. Li, S.; Dong, S.; Xu, W.; Tu, S.; Yan, L.; Zhao, C.; Ding, J.; Chen, X. Antibacterial Hydrogels. Adv. Sci. 2018, 5, 1700527.

3. Xuewen, D.; Zhou, J.; Shi, J.; Xu, B. Supramolecular Hydrogelators and Hydrogels: From Soft Matter to Molecular Biomaterials. Chem. Rev. 2015, 115, 13165-13307.

4. He, M.; Wang, Q.; Zhang, J.; Zhao, W.; Zhao, C. Substrate-Independent AgNanoparticle-Loaded Hydrogel Coating with Regenerable Bactericidal and Thermoresponsive Antibacterial Properties. ACS Appl. Mater. Interfaces 2017, 9, 4478244791.

5. Tang, Q.; Plank, T. N.; Zhu, T.; Yu, H.; Ge, Z.; Li, Q.; Li, L.; Davis, J. T.; Pei, H. SelfAssembly of Metallo-Nucleoside Hydrogels for Injectable Materials that Promote Wound Closure. ACS Appl. Mater. Interfaces 2019, 11, 19743- 19750. 
6. Lakes, A. L.; Peyyala, R.; Ebersole, J. L.; Puelo, D. A.; Hilt, J. Z.; Dziubla, T. D. Synthesis and Characterization of an Antibacterial Hydrogel Containing Covalently Bound Vancomycin. Biomacromolecules 2014, 15, 3009- 3018.

7. Xing, B.; Yu, C.-W.; Chow, K.-H.; Ho, P.-L.; Fu, D.; Xu, B. Hydrophobic Interaction and Hydrogen Bonding Cooperatively Confer a Vancomycin Hydrogel: A Potential Candidate for Biomaterials. J. Am. Chem. Soc. 2002, 124, 14846- 14847.

8. Baral, A.; Roy, S.; Ghosh, S.; Hermida-Merino, D.; Hamley, I. W.; Banerjee, A. A Peptide-Based Mechano-sensitive, Proteolytically Stable Hydrogel with Remarkable Antibacterial Properties. Langmuir 2016, 32, 1836- 1845.

9. Lei, K.; Wang, K.; Sun, Y.; Zheng, Z.; Wang, X. Rapid-Fabricated and Recoverable Dual-Network Hydrogel with Inherently Antibacterial Abilities for Potential Adhesive Dressings. Adv. Funct. Mater. 2020, 31, 2008010.

10. Zhong, Y.; Xiao, H.; Seidi, F.; Jin, Y. Natural Polymer-Based Antimicrobial Hydrogels Without Synthetic Antibiotics as Wound Dressings. Biomacromolecules 2020, 21, 29833006.

11. Draper, E. R.; Adams, D. J. Low-Molecular-Weight Gels: The State of the Art. Chem. 2017, 3, 390-410.

12. Raeburn, J.; Cardoso, A. Z.; Adams, D. J. The Importance of the Self-Assembly Process to Control Mechanical Properties of Low Molecular Weight Hydrogels. Chem. Soc. Rev. 2013, 42, 5143-5156.

13. Yan, J.; Ji, Y.; Huang, M.; Li, T.; Liu, Y.; Lü, S.; Liu, M. Nucleobase-Inspired SelfAdhesive and Inherently Antibacterial Hydrogel for Wound Dressing. ACS Materials Lett. 2020, 2, 1375-1380.

14. Sreenivasachary, N.; Lehn, J.-M. Structural Selection in G-Quartet-Based Hydrogels and Controlled Release of Bioactive Molecules. Chem.-Asian J. 2008, 3, 134-139.

15. Nandi, N.; Gayen, K.; Ghosh, S.; Bhunia, D.; Kirkham, S.; Sen, S. K.; Ghosh, S.; Hamley, I. W.; Banerjee, A. Amphiphilic Peptide-Based Supramolecular, Noncytotoxic, Stimuli-Responsive Hydrogels with Antibacterial Activity. Biomacromolecules 2017, 18, $3621-3629$.

16. Fichman, G.; Gazit, E. Self-Assembly of Short Peptides to Form Hydrogels: Design of Building Blocks, Physical Properties and Technological Applications. Acta Biomater. 2014, 10, 1671- 1682 .

17. Wang, H.; Yang, Z. Short-Peptide-Based Molecular Hydrogels: Novel Gelation Strategies and Applications for Tissue Engineering and Drug Delivery. Nanoscale 2012, 4, 52595267.

18. Liu, X., Sun, X., and Liang, G. Peptide-based Supramolecular Hydrogels for Bioimaging Applications. Biomater. Sci. 2021, 9, 315-327.

19. Ryan, D. M.; Nilsson, B. L. Self-assembled amino acids and dipeptides as noncovalent hydrogels for tissue engineering. Polym. Chem. 2012, 3, 18- 33.

20. Peters, G. M.; Davis, J. T. Supramolecular Gels Made from Nucleobase, Nucleoside and Nucleotide Analogs. Chem. Soc. Rev. 2016, 45, 3188- 3206.

21. Buerkle, L. E.; Li, Z.; Jamieson, A. M.; Rowan, S. J. Tailoring the Properties of Guanosine-Based Supramolecular Hydrogels. Langmuir 2009, 25, 8833- 8840. 
22. Davis, J. T.; Spada, G. P. Supramolecular Architectures Generated by Self-assembly of Guanosine Derivatives. Chem. Soc. Rev. 2007, 36, 296- 313.

23. Rotaru, A.; Pricope, G.; Plank, T. N.; Clima, L.; Ursu, E. L.; Pinteala, M.; Davis, J. T.; Barboiu, M. G-Quartet hydrogels for effective cell growth applications. Chem. Commun. 2017, 53, 12668- 12671.

24. Adhikari, B.; Shah, A.; Kraatz, H.-B. Self-Assembly of Guanosine and Deoxy-Guanosine into Hydrogels: Monovalent Cation Guided Modulation of Gelation, Morphology and Self-Healing Properties. J. Mater. Chem. B 2014, 2, 4802- 4810.

25. Rowan, S. J.; Cantrill, S. J.; Cousins, G. R. L.; Sanders, J. K. M.; Stoddart, J. F. Dynamic Covalent Chemistry. Angew. Chem., Int. Ed. 2002, 41, 898- 952.

26. Guan, Y.; Zhang, Y. Boronic Acid-Containing Hydrogels: Synthesis and Their Applications. Chem. Soc. Rev. 2013, 42, 8106-8121.

27. Heleg-Shabtai, V.; Aizen, R.; Orbach, R.; Aleman-Garcia, M. A.; Willner, I. GossypolCross-Linked Boronic Acid-Modified Hydrogels: a Functional Matrix for the Controlled Release of an Anticancer Drug. Langmuir 2015, 31, 2237- 2242.

28. Brooks, W. L. A.; Sumerlin, B. S. Synthesis and applications of boronic acid-containing polymers: From materials to medicine. Chem. Rev. 2016, 116, 1375- 1397.

29. Li, Y.; Yang, L.; Zeng, Y.; Wu, Y.; Wei, Y.; Tao, L. Self-Healing Hydrogel with a Double Dynamic Network Comprising Imine and Borate Ester Linkages. Chem. Mater. 2019, 31, 5576- 5583.

30. Ghosh, T.; Biswas, A.; Gavel, P. K.; Das, A. K. Engineered Dynamic Boronate EsterMediated Self-Healable Biocompatible G-Quadruplex Hydrogels for Sustained Release of Vitamins. Langmuir 2020, 36, 1574- 1584.

31. Chatterjee, S.; Anslyn, E. V.; Bandyopadhyay, A. Boronic acid based dynamic click chemistry: recent advances and emergent applications. Chem. Sci. 2021, 12, 1585- 1599.

32. António, J. P. M.; Russo, R.; Carvalho, C. P.; Cal, P. M. S. D.; Gois, P. M. P. Boronic acids as building blocks for the construction of therapeutically useful bioconjugates. Chem. Soc. Rev. 2019, 48, 3513.

33. Elsherif, M.; Hassan, M. U.; Yetisen, A. K.; Butt, H. Glucose Sensing with Phenylboronic Acid Functionalized Hydrogel-Based Optical Diffusers. ACS Nano 2018, 12, 2283-2291.

34. Smithmyer, M. E.; Deng, C. C.; Cassel, S. E.; LeValley, P. J.; Sumerlin, B. S.; Kloxin, A. M. Self-healing boronic acid-based hydrogels for 3D co-cultures. ACS Macro Lett. 2018, 7, 1105- 1110 .

35. Lee, A. L. Z.; Voo, Z. X.; Chin, W.; Ono, R. J.; Yang, C.; Gao, S.; Hedrick, J. L.; Yang, Y. Y. Injectable Coacervate Hydrogel for Delivery of Anticancer Drug-Loaded Nanoparticles in Vivo. ACS Appl. Mater. Interfaces 2018, 10, 13274-13282.

36. Belowich, M. E.; Stoddart, J. F. Dynamic imine chemistry. Chem. Soc. Rev. 2012, 41, 2003-2024.

37. Boehnke, N.; Cam, C.; Bat, E.; Segura, T.; Maynard, H. D. Imine Hydrogels with Tunable Degradability for Tissue Engineering. Biomacromolecules 2015, 16, 21012108. 
38. Li, Y.; Liu, Y.; Ma, R.; Xu, Y.; Zhang, Y.; Li, B.; An, Y.; Shi, L. A G -Quadruplex Hydrogel via Multicomponent Self-Assembly: Formation and Zero-Order Controlled Release. ACS Appl. Mater. Interfaces 2017, 9, 13056.

39. Biswas, A.; Ghosh, T.; Gavel, P. K.; Das, A. K. PEG Functionalized Stimuli Responsive Self-Healable Injectable Dynamic Imino-boronate G-quadruplex Hydrogel for the Delivery of Doxorubicin. ACS Appl. Bio Mater. 2020, 3, 1052- 1060.

40. Meng, H.; Xiao, P.; Gu, J. C.; Wen, X. F.; Xu, J.; Zhao, C. Z.; Zhang, J. W.; Chen, T. Self-Healable Macro-/Microscopic Shape Memory Hydrogels Based on Supramolecular Interactions. Chem. Commun. 2014, 50, 12277-12280.

41. Pettignano, A.; Grijalvo, S.; Häring, M.; Eritja, R.; Tanchoux, N.; Quignard, F.; Díaz, D. D. Boronic Acid-Modified Alginate Enables Direct Formation of Injectable, Self-Healing and Multistimuli-Responsive Hydrogel. Chem. Commun. 2017, 53, 3350.

42. Guan, A.-j.; Zhang, X.-F.; Sun, X.; Li, Q.; Xiang, J.-F.; Wang, L.-X.; Lan, L.; Yang, F.M.; Xu, S.-J.; Guo, X.-M.; Tang, Y.-L. Ethyl substitutive Thioflavin T as a highlyspecific fluorescence probe for detecting G-quadruplex structure. Sci. Rep. 2018, 8, 2666.

43. Ajayaghosh, A.; Vijayakumar, C.; Varghese, R.; George, S. J. Cholesterol-aided supramolecular control over chromophore packing: twisted and coiled helices with distinct optical, chiroptical, and morphological features. Angew. Chem., Int. Ed. 2006, 45, 456- 460 .

44. Peters, G. M.; Skala, L. P.; Plank, T. N.; Hyman, B. J.; Manjunatha Reddy, G. N.; Marsh, A.; Brown, S. P.; Davis, J. T. A G4·K+ Hydrogel Stabilized by an Anion. J. Am. Chem. Soc. 2014, 136, 12596- 12599.

45. Biswas, A.; Malferrari, S.; Kalaskar, D. M.; Das, A. K. Arylboronate Esters Mediated Self-Healable and Biocompatible Dynamic G-Quadruplex Hydrogels as Promising 3DBioinks. Chem. Commun. 2018, 54, 1778- 1781.

46. Arnal-Hérault, C.; Pasc, A.; Michau, M.; Cot, D.; Petit, E.; Barboiu, M. Functional GQuartet Macroscopic Membrane Films. Angew. Chem., Int. Ed. 2007, 46, 8409-8413.

47. Arnal-Hérault, C.; Banu, A.; Barboiu, M.; Michau, M.; van der Lee, A. Amplification and Transcription of the Dynamic Supramolecular Chirality of the Guanine Quadruplex. Angew. Chem., Int. Ed. 2007, 119, 4346-4350.

48. Peters, G. M.; Skala, L. P.; Plank, T. N.; Oh, H.; Manjunatha Reddy, G. N.; Marsh, A.; Brown, S. P.; Raghavan, S. R.; Davis, J. T. G4-Quartet·M+ Borate Hydrogels. J. Am. Chem. Soc. 2015, 137, 5819- 5827.

49. Ghosh, T.; Biswas, A.; Bhowmik, S.; Das, A. K. Pt Nanoparticles Supported on a Dynamic Boronate Ester-Based G-quadruplex Hydrogel as a Nanoreactor. Chem. - Asian J. 2021, 16, 215-223.

50. Gavel, P. K.; Dev, D.; Parmar, H. S.; Bhasin, S.; Das, A. K. Investigations of PeptideBased Biocompatible Injectable Shape-Memory Hydrogels: Differential Biological Effects on Bacterial and Human Blood Cells. ACS Appl. Mater. Interfaces 2018, 10, $10729-10740$.

51. Wang, J.; Wang, L.; Wu, C.; Pei, X.; Cong, Y.; Zhang, R.; Fu, J. Antibacterial Zwitterionic Polyelectrolyte Hydrogel Adhesives with Adhesion Strength Mediated by Electrostatic Mismatch. ACS Appl. Mater. Interfaces 2020, 12, 46816- 46826. 